\title{
MCE activities and malathion resistances in field populations of the Australian sheep blowfly (Lucilia cuprina)
}

\author{
KERRIE-ANN SMYTH, * THOMAS M. BOYCE, ROBYN J. RUSSELL \\ \& JOHN G. OAKESHOTT \\ CSIRO Division of Entomology, GPO Box 1700, Canberra, ACT 2601, Australia
}

\begin{abstract}
Malathion resistance has been shown to be the result of a single point mutation in the $L c \alpha \mathrm{E} 7$ gene in four independently isolated chromosomes of Lucilia cuprina. The resultant amino acid substitution specifies high malathion carboxylesterase (MCE) activity. We have assayed MCE activities and resistance to malathion in three sets of field-derived samples, two sets of isogenic lines and five mass populations, and show that resistance to malathion in these samples is associated with high MCE activity in both sets of isogenic lines and four of the five mass populations. Additional mechanisms contributing to MCE activity or malathion resistance may be present in one of the mass populations. A second point mutation in $L c \alpha \mathrm{E} 7$ is responsible for conferring diazinon resistance by encoding an increased organophosphate (OP) hydrolase activity. We also assayed diazinon resistances from the same three samples and show that diazinon and malathion resistances were in complete disequilibrium, with two exceptions. One exception involves the mass population with additional resistance mechanism(s) and the other involves three isogenic lines that are resistant to both insecticides. The molecular data for these lines suggest that they carry a duplication of the $L c \alpha \mathrm{E} 7$ gene.
\end{abstract}

Keywords: carboxylesterase, Diptera, genetic variation, malathion, organophosphate, resistance.

\section{Introduction}

Resistance to organophosphates (OPs) in Lucilia cuprina was first recorded in 1965 as resistance to diazinon (Shanahan \& Hart, 1966). The frequency of diazinon resistant flies in the field was reported to be $97 \%$ in 1981 (Hughes, 1981) and it has remained high since then (Levot, 1995). In contrast to diazinon resistance, the frequency of malathion resistant flies in the field is generally low, approximately 11\% (Hughes et al., 1984; Levot, 1995). However, given that malathion has not been directly used to control L. cuprina, this level of resistance is a little surprising.

Resistance to diazinon and malathion is primarily the result of two point mutations in a carboxylesterase gene on the fourth chromosome ( $L c \alpha \mathrm{E} 7)$, encoding both OP hydrolase and malathion carboxylesterase (MCE) activities (Campbell et al., 1997; Newcomb et al., 1997; 1998a). The OP hydrolase confers resistance to diazinon

*Correspondence. Present address: Institute of Cellular and Molecular Biology, University of Texas at Austin, Molecular Biology Building, Mail Code A4800, Austin, Texas 78712-1095, U.S.A. E-mail: ksmyth@mail.utexas.edu and many other OPs, whereas high MCE activity confers resistance to malathion and a small number of OPs with carboxylester bonds (Raftos, 1986; Campbell et al., 1997; 1998b). Diazinon resistance in L. cuprina has traditionally been measured by electrophoretic analysis using $\alpha$-napthylacetate as a substrate, and assaying for esterase 3 (E3) activity. The resistance conferring form of E3, the OP hydrolase, lacks any measurable carboxylesterase activity and appears as 'nonstaining' on native polyacrylamide gels (PAGE; McKenzie et al., 1992). The loss of carboxylesterase activity is caused by a single point mutation in the $L c \alpha \mathrm{E} 7$ gene resulting in a Gly ${ }^{137}-\mathrm{Asp}$ amino acid substitution (Newcomb et al., 1997), which results in the increase of OP hydrolase and confers diazinon resistance (Campbell et al., 1997; 1998a).

Recent evidence shows that malathion resistance is also conferred by a mutation in the $L c \alpha \mathrm{E} 7$ gene. Campbell et al. (1998a) found that a $\operatorname{Trp}^{251}$-Leu amino acid substitution in E3 is responsible for high MCE activity in four malathion resistant lines of $L$. cuprina. Malathion resistance is caused by increased specificity of E3 for malathion and resistance to inhibition by malaoxon (the active desulphurated form of malathion). 
Biochemical analysis of the MCE activity revealed three different forms of MCE (Smyth et al., 1996). High MCE activity is caused by the $\operatorname{Trp}^{251}$-Leu amino acid substitution, which increases MCE activity sixfold, intermediate MCE activity is caused by the wild-type enzyme and low MCE activity is the result of the Gly ${ }^{137}$-Asp amino acid substitution, which greatly reduces the enzyme's carboxylesterase activities (Campbell et al., 1998a). The high phenotype is 1000 -fold more resistant to malathion than either of the other two phenotypes, despite the small difference in activities between the high and intermediate types (high has 1.6-fold greater activity than intermediate MCE activity; Smyth et al., 1994).

We have assayed MCE activities and malathion resistance from three field-derived samples, two sets of lines made isogenic for chromosome 4 and one set consisting of five mass populations, to determine the contribution of the $\operatorname{Trp}^{251}$-Leu amino acid substitution in E3 to high MCE activity and malathion resistance in the field. We also explore whether double resistance to both malathion and diazinon can be the result of the two mutations occurring in the $L c \alpha \mathrm{E} 7$ gene.

\section{Materials and methods}

\section{Strains}

The first sample consists of 34 isogenic lines: four (RM2-6, Q4, LS2 and RM8) from an earlier study of seven strains (Smyth et al., 1994) and 30 new lines generated the same way. Briefly, wild-type males were crossed with three virgin females heterozygous for a multiply inverted fourth chromosome balancer $[\operatorname{In}(4) 6+8+12 S h \mathrm{gl} / \mathrm{sv} \mathrm{ra} \mathrm{tg} \mathrm{gl}]$. Individual $\mathrm{F}_{1}$ males heterozygous for the balancer were backcrossed to three virgin balancer strain females and the $\mathrm{G}_{2}$ progeny $[\operatorname{In}(4) 6+8+12 S h \mathrm{gl} /++++]$ were intercrossed in single-pair matings. Wild-type $\mathrm{G}_{3}$ progeny from each $G_{2}$ cross were then intercrossed to establish the isogenic lines. These 30 new and the four original isogenic lines are termed the unscreened lines because they were not screened with malathion during the extraction procedure. Two low MCE and one intermediate MCE strain from the original survey are not isogenic lines and have been omitted from the statistical analysis of these data (Sh, Dua-91 and sv bu ra).

The second sample contains 20 isogenic lines generated by the same procedure except that males used as parents in the $G_{0}$ and $G_{1}$ generations had survived a dose of $0.2 \% \mathrm{w} / \mathrm{v}(2 \mu \mathrm{g} / \mu \mathrm{L})$ malathion, which discriminates against malathion susceptible individuals (Hughes et al., 1984). Malathion in acetone $(1 \mu \mathrm{L})$ was topically applied to the upper thorax of each parental male, 3 to 5 days after eclosion. These 20 isogenic lines are termed the screened lines.

The five mass populations were: Finley (collected 1991); Flinders Island (collected 1988); LBB (collected before 1960); Llandillo (collected 1991); Murrumbateman (collected 1977; Foster et al., 1978). All strains were maintained in the laboratory without exposure to insecticides. The distribution of collection sites for the isogenic lines and mass populations is illustrated in Fig. 1.

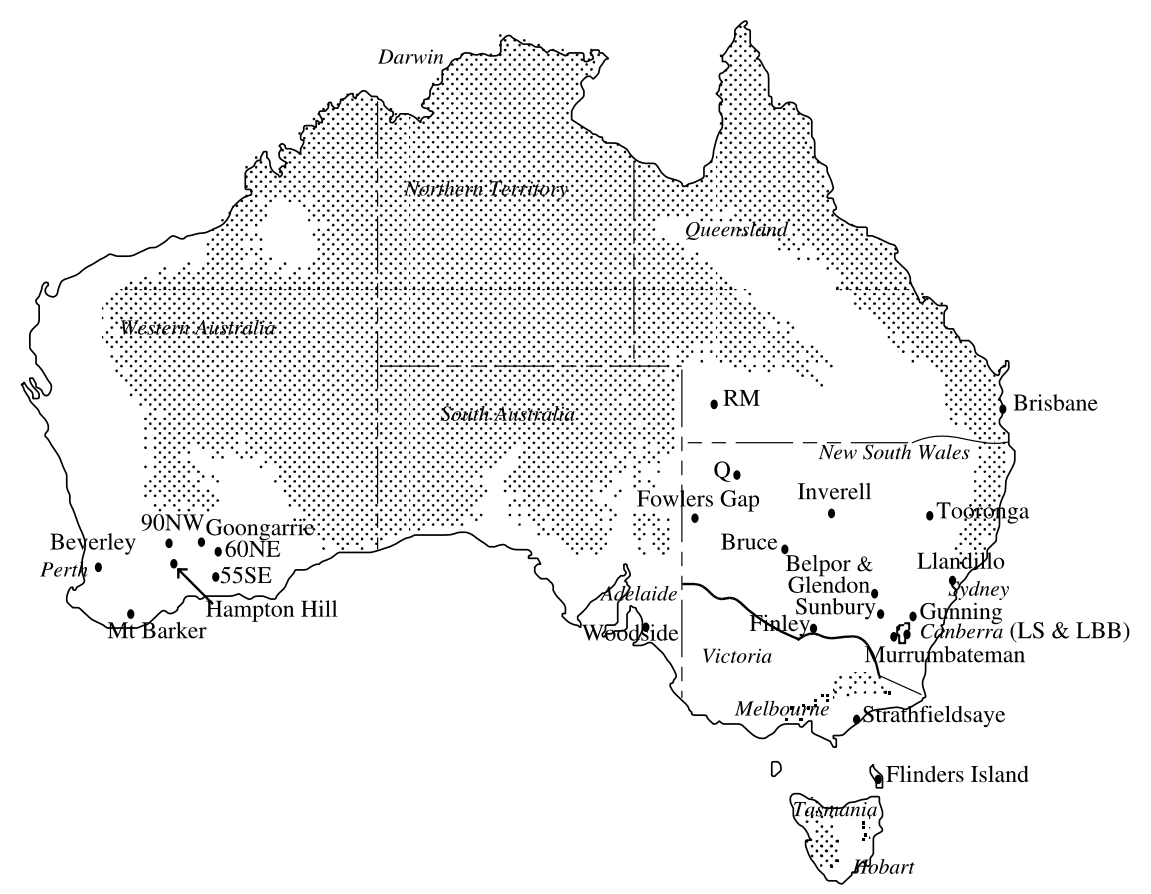

Fig. 1 Map of Australia showing the collection sites of the strains of Lucilia cuprina from which the isogenic lines and mass populations were derived. The unshaded area represents the regions of Australia where sheep are reared (Norris, 1990). The states and capital cities are italicized. 
Adult flies were maintained on a diet of sugar and water and given a protein feed prior to oviposition. Flies were assayed within the first week of eclosion.

\section{Biochemical assays}

MCE activity was assayed using the radiometric partition method previously described by Whyard et al. (1994). Individual flies were homogenized in $600 \mu \mathrm{L}$ MCE homogenization buffer (10 $\mathrm{mm}$ imidazole $\mathrm{HCl}$, $\mathrm{pH} 7.0,1 \%$ Triton $\mathrm{X}-100(\mathrm{v} / \mathrm{v}), 1 \mathrm{~mm}$ phenylthiourea and $1 \mathrm{~mm}$ dithiothreitol) and immediately assayed for MCE activity. Activities were calculated from aliquots of $15 \mu \mathrm{L}$ of homogenate for lines expressing high or intermediate MCE activities, and $75 \mu \mathrm{L}$ for low lines and individuals from the mass populations. Protein concentrations were determined using the method of Bradford (1976) with ovalbumin as the standard.

The MCE enzymes in adults of six screened isogenic lines were tested for their susceptibility to diagnostic inhibitors that distinguish the low, intermediate and high MCE phenotypes (Smyth et al., 1996): paraoxon $\left(2 \times 10^{-7} \mathrm{M}\right)$ and triphenylphosphate (TPP; $\left.3 \times 10^{-6} \mathrm{M}\right)$. Fifteen microlitres of homogenate from each line was preincubated with inhibitor for $30 \mathrm{~min}$ at $25^{\circ} \mathrm{C}$ before assaying for MCE activity. Control assays were preincubated with an equal volume of inhibitor solvent, ethanol.

\section{Toxicology}

Dose-mortality relationships were determined for a representative subset of the 54 isogenic lines. One microlitre of the appropriate concentration of OP dissolved in acetone was topically applied to the upper thorax of 3-5 day old adults. Twenty-five females were tested and mortality was recorded after $24 \mathrm{~h}$. Malathion was applied to the unscreened lines at seven concentrations ranging from 0 to $0.072 \%(\mathrm{w} / \mathrm{v})$ and to the screened lines at six concentrations ranging from 0 to $40 \%(\mathrm{w} / \mathrm{v})$. Diazinon was applied to both the unscreened and screened lines at six concentrations ranging from 0 to $0.04 \%(\mathrm{w} / \mathrm{v})$. Control adult flies were tested with acetone only. The concentration of each insecticide at which $50 \%$ of the flies died was estimated by probit analysis (Finney, 1971) as implemented in SAS, version 6.03 (SAS, 1988).

Discriminating dose assays for malathion were carried out on adults from four additional screened lines (25 flies of each sex per line) and for both malathion and diazinon in the five mass populations (50 flies of each sex per population). The doses were chosen to discriminate susceptible homozygotes from resistant heterozygotes and homozygotes: $0.2 \%(\mathrm{w} / \mathrm{v})$ for malathion (Hughes et al., 1984) and $0.01 \%(\mathrm{w} / \mathrm{v})$ for diazinon (McKenzie et al., 1980), applied topically as described above. Control flies were treated with acetone and mortality was recorded after $24 \mathrm{~h}$.

Diazinon larval toxicology was determined using a dose that again discriminates susceptible homozygotes from resistant heterozygotes or homozygotes (McKenzie et al., 1992). Two hundred first instar larvae were placed on meat meal media containing $0.0003 \%$ diazinon and the number of emerging flies counted to determine larval resistance. Control assays were performed without diazinon, and three replicates of each assay were performed.

\section{Genetics}

Crosses between several isogenic lines were set up to determine the dominance relationships among the high, intermediate and low MCE specific activities. Virgin females from one MCE specific activity line were crossed with males from a different MCE specific activity line (the lines used and crosses established are described in the Results). For each cross, $20 \mathrm{~F}_{1}$ individuals of each sex were assayed for MCE specific activity using $15 \mu \mathrm{L}$ of homogenate per individual.

\section{Results}

\section{Unscreened isogenic lines}

MCE specific activities for 28 of the 30 new unscreened lines ranged from $4.0 \pm 0.5$ to $14.2 \pm 0.6$ pmole malathion $/ \mathrm{min} / \mathrm{mg}$ (Fig. 2a) and were comparable to that of the two low MCE lines $(6.6 \pm 0.2$ and $9.6 \pm 0.8)$ previously described in Smyth et al. (1994). There was significant variation in activity among all low MCE activity isogenic lines $\left(F_{29,200}=7.45, P \leq 0.0001\right)$. No obvious correlate explained the variation (e.g. geographical origin, time of collection). The remaining two unscreened lines fell into the intermediate MCE group (Fig. 2a). The values for the two new intermediate MCE lines $(112 \pm 5$ and $138 \pm 5$ pmole malathion $/ \mathrm{min} / \mathrm{mg})$ were similar to the previously described intermediate line $(139 \pm 3)$. There was no significant variation of activities among these isogenic lines $\left(F_{2,28}=0.91\right.$, $P>0.05)$. Combining the data for all the new lines and the four isogenic lines from the initial survey (RM26, Q4, LS2 and RM8 on Fig. 2a) yielded frequencies of $88 \pm 2 \%$ for low MCE, $9 \pm 2 \%$ for intermediate MCE and $3 \pm 1 \%$ for high MCE activity lines.

$\mathrm{LD}_{50}$ values for malathion in a subsample of five unscreened isogenic lines (three low MCE activity and two intermediate MCE activity lines) were similar to those previously reported (Smyth et al., 1994). Adult 



Fig. 2 Distribution of MCE specific activity (pmole malathion $\mathrm{min} /$ $\mathrm{mg} \pm \mathrm{SE}$ ). Low MCE activity lines are indicated by clear bars, intermediate MCE activity lines by grey bars and the high MCE activity line by the black bar. The asterisks indicate the four isogenic lines of Lucilia cuprina originally described in Smyth et al. (1994). (a) Unscreened isogenic lines. (b) Screened isogenic lines.
$\mathrm{LD}_{50}$ values differed 1000-fold between the previously described high MCE activity, malathion resistant line and the low and intermediate MCE activity, malathion susceptible lines (Table 1). The $\mathrm{LD}_{50}$ values for adults of diazinon resistant lines were approximately eightfold higher than those of diazinon susceptible lines. The levels of resistance to the two insecticides concur with those reported earlier with other strains.

Larvae from the RM8, LS2 and RM2-6 lines were also tested for resistance to diazinon (data not shown). The RM2-6 larvae were resistant, the RM8 and LS2 larvae were not, mirroring the adult resistance phenotypes of the three lines.
The dominance relationships between the low, intermediate and high MCE activities were determined to allow assignment of these activities within the five nonisogenic mass populations. Dominance relationships for MCE activity among six of the unscreened isogenic lines were the same as those evident among the strains used previously to map MCE activity (Fig. 3; Smyth et al., 1994). The crosses of the three low MCE lines with the high MCE line yielded midparental values in the $\mathrm{F}_{1}\left(t_{155}=0.64, P \geq 0.05\right)$. However, crosses between two of the low and two of the intermediate MCE lines gave $F_{1}$ activities significantly less than midparental $\left(t_{155}=3.14, \quad P \leq 0.05\right)$, as did crosses between two intermediate MCE lines and the high MCE line 
Table 1 Female malathion and diazinon $\mathrm{LD}_{50}$ values $(\% \mathrm{w} / \mathrm{v})$ for the isogenic lines of Lucilia cuprina

\begin{tabular}{|c|c|c|c|c|c|c|}
\hline \multirow[b]{2}{*}{ Line } & \multicolumn{3}{|c|}{ Malathion } & \multicolumn{3}{|c|}{ Diazinon } \\
\hline & $\mathrm{LD}_{50}$ & $\mathrm{LD}_{10}, \mathrm{LD}_{90}$ & Resistance status & $\mathrm{LD}_{50}$ & $\mathrm{LD}_{10}, \mathrm{LD}_{90}$ & Resistance status \\
\hline \multicolumn{7}{|c|}{ (a) Unscreened isogenic lines } \\
\hline RM2-6† & 0.04 & $0.02,0.09$ & $\mathrm{~S} \ddagger$ & 0.04 & $0.02,0.06$ & $\mathrm{R}$ \\
\hline Inverrell 22 & 0.03 & $0.02,0.05$ & $\mathrm{~s}$ & 0.03 & $0.02,0.03$ & $\mathrm{R}$ \\
\hline Llandillo 103 & 0.05 & $0.04,0.07$ & $\mathrm{~S}$ & 0.02 & $0.01,0.03$ & $\mathrm{R}$ \\
\hline Sunbury $5.2 \dagger$ & 0.02 & $0.01,0.03$ & $\mathrm{~S}$ & 0.02 & $0.01,0.04$ & $\mathrm{R}$ \\
\hline $\mathrm{LS} 2 \dagger$ & 0.05 & $0.03,0.07$ & $\mathrm{~S}$ & 0.004 & $0.002,0.007$ & $\mathrm{~S}$ \\
\hline LBB 101 & 0.05 & $0.04,0.07$ & $\mathrm{~S}$ & 0.005 & $0.003,0.006$ & $\mathrm{~S}$ \\
\hline Llandillo 104 & 0.04 & $0.03,0.06$ & $\mathrm{~S}$ & 0.005 & $0.004,0.006$ & $\mathrm{~S}$ \\
\hline RM8† & 35 & 24,50 & $\mathrm{R}$ & 0.008 & $0.006,0.01$ & $\mathrm{~S}$ \\
\hline \multicolumn{7}{|c|}{ (b) Screened isogenic lines } \\
\hline Beverly 4.2 & 7 & 5,10 & $\mathrm{R}$ & NT§ & NT & $\mathrm{S}$ \\
\hline Beverly 6.2 & 5 & 3,9 & $\mathrm{R}$ & NT & NT & $\mathrm{S} \boldsymbol{\top}$ \\
\hline Hampton Hill 6.1 & 15 & 12,19 & $\mathrm{R}$ & 0.002 & $0.001,0.003$ & $\mathrm{~S}$ \\
\hline Hampton Hill 6.2 & 9 & 7,11 & $\mathrm{R}$ & 0.003 & $0.002,0.004$ & $\mathrm{~S}$ \\
\hline Tooronga 2.2 & 23 & 15,36 & $\mathrm{R}$ & 0.06 & $0.02,0.08$ & $\mathrm{R}$ \\
\hline Tooronga 2.3 & 25 & 16,41 & $\mathrm{R}$ & NT & NT & $\mathrm{S}$ \\
\hline Woodside 5.2 & 17 & 13,23 & $\mathrm{R}$ & 0.005 & $0.003,0.007$ & $\mathrm{~S}$ \\
\hline 55SE 14.1 & 12 & 5,27 & $\mathrm{R}$ & NT & NT & $\mathrm{R} \boldsymbol{\top}$ \\
\hline 60NE 1.1 & 7 & 4,13 & $\mathrm{R}$ & 0.013 & $0.007,0.02$ & $\mathrm{R}$ \\
\hline
\end{tabular}

$\dagger$ Originally described in Smyth et al. (1994).

$\$$ Susceptible (S) vs. Resistant (R).

$\S$ Not Tested.

$\uparrow$ Resistance status determined by discriminating dose.

$\left(t_{155}=10.19, P \leq 0.001\right) . \mathrm{F}_{1}$ values for the latter cross overlap that of intermediate MCE homozygotes $(121 \pm 6$ vs. $129 \pm 7$ pmole malathion $/ \mathrm{min} / \mathrm{mg}$, respectively). There were no differences among any of the reciprocal crosses.

Application of Duncan's multiple range test (Duncan, 1975) to the total data on MCE specific activity for all the unscreened lines and the crosses among them revealed only four groups of phenotypes. Those that were not significantly different at $P<0.05$ were intermediate homozygotes, high/intermediate heterozygotes and high/low MCE heterozygotes. The high MCE homozygotes, intermediate/low MCE heterozygotes and low MCE homozygotes were all different from one another and from the above group.

\section{Screened isogenic lines}

The screened isogenic lines were created to recover as many different malathion resistance alleles as possible within the field populations from which these flies were collected. There was significant variation among the MCE activities of the 20 screened lines $(164.8 \pm 7.2$ to $335.2 \pm 6.9$ pmole malathion $/ \mathrm{min} / \mathrm{mg} ; F_{19,197}=25.5$, $P \leq 0.001)$ but even the lowest activity levels were above those for the three intermediate activity lines (Fig. 2b).
The MCE activities of six of the screened lines (Beverly 6.2, Hampton Hill 6.1, Hampton Hill 6.2, Tooronga 2.2, Woodside 5.2 and 60NE 1.1; see Fig. 2b) were tested for susceptibility to the diagnostic inhibitors TPP and paraoxon. Previous analysis of three of the original unscreened lines had shown that $3 \times 10^{-6} \mathrm{M}$ TPP and $2 \times 10^{-7} \mathrm{M}$ paraoxon distinguish the different MCE activities and completely inhibit high MCE activity (Smyth et al., 1996). Both TPP and paraoxon also completely inhibited the MCE activities of all six screened lines, suggesting that they were similar to that of RM8.

The $\mathrm{LD}_{50}$ values indicate that all the screened lines were resistant to malathion, as expected (Table 1). The differences in degrees of resistance to malathion correlate closely with the variation in MCE activity among these lines (linear regression, $F_{1,7}=6.50, P \leq 0.01$ ). The variation in resistance is greater than the variation in activity (fivefold vs. twofold) but is still small compared to the difference between resistance and susceptibility. Three of the nine lines tested were also resistant to diazinon: Tooronga 2.2, 55SE 14.1 and $60 \mathrm{NE} 1.1 .60 \mathrm{NE}$ 1.1 showed substantially less resistance than Tooronga 2.2 (diazinon resistance in 55SE 14.1 was determined by discriminating dose), and than the resistant unscreened lines (1.5- to 5-fold less resistance). 
(a)



(b)

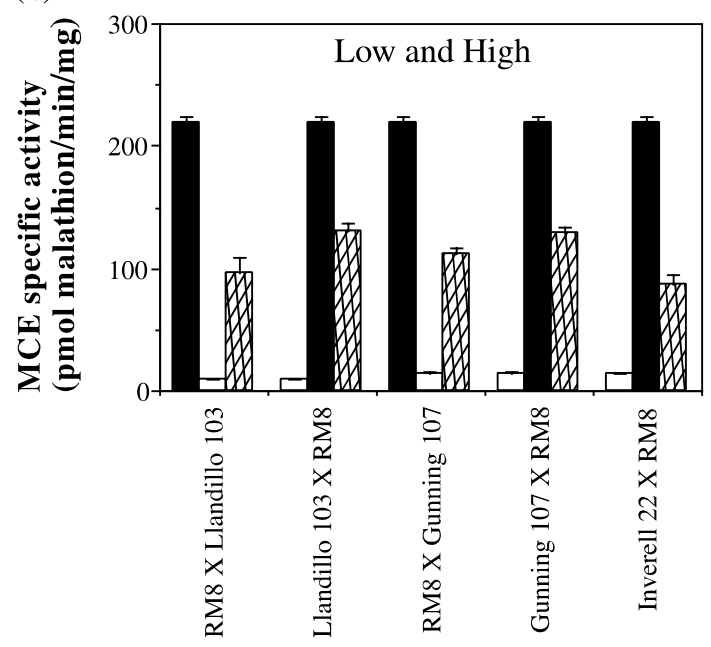

(c)

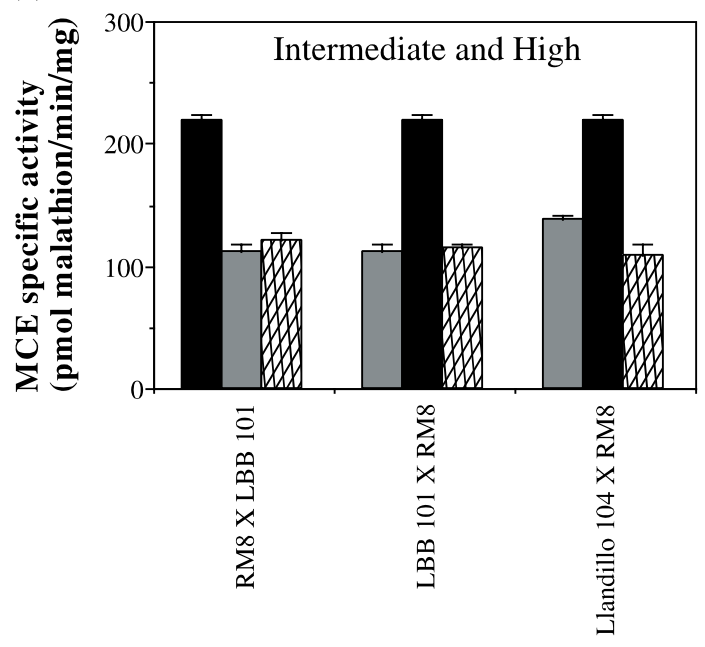

Fig. 3 Dominance relationships between the low, intermediate and high MCE phenotype of Lucilia cuprina. The MCE specific activity $( \pm \mathrm{SE})$ is given for each parental isogenic and $F_{1}$ line. Low MCE activity lines are indicated by clear bars, intermediate MCE activity lines by grey bars, high MCE activity line by black bars and the $F_{1}$ progeny by hatched bars. The female parent is listed first in each cross. (a) Low and intermediate MCE phenotypes. (b) Low and high MCE phenotypes.

(c) High and intermediate MCE phenotypes.

Larvae of the Woodside 5.2 line were susceptible to diazinon and those of Tooronga 2.2 were resistant (data not shown), consistent with the respective adult resistance phenotypes of these lines.

\section{Mass populations}

MCE activities were determined for 100 individual flies from each of five mass populations (Fig. 4). The distribution of activities was clearly discontinuous in each population. One narrow peak in each population corresponded to the activity of low MCE homozygotes. The frequency of these individuals was high in Finley $(86 \%)$, the most recently collected population (1991), and comparable to frequencies of low MCE chromosomes in the unscreened lines. However, the frequencies of the putative low MCE homozygotes were substantially lower in the other four populations, ranging from $9 \%$ in LBB to $42 \%$ in Murrumbateman.

All individuals from each population not classified as low MCE homozygotes had specific activities in the range compatible with the various intermediate and high MCE genotypes found in the isogenic lines. Precise frequencies of intermediate and high MCE homozygotes in the mass populations cannot be calculated because of the evidence from the isogenic lines for complex dominance relationships in heterozygotes. However, most of the 'nonlow' MCE individuals from four of the populations lay in the range of activities for the intermediate MCE homozygotes and the intermediate/ high and low/intermediate MCE heterozygotes, whereas the majority of the 'nonlow' MCE individuals from the Finley population had higher values, in the range previously ascribed to high MCE homozygotes. Correcting for variations in protein content did not significantly change the data.

The proportion of flies susceptible to malathion or diazinon in the five mass populations was estimated as the frequency of individuals that did not survive a dose of malathion designed to kill susceptible homozygotes. The frequency of malathion susceptible flies varied from $73 \%$ in the Finley population to $96 \%$ in Flinders Island (Table 2), and was higher in four populations than the 


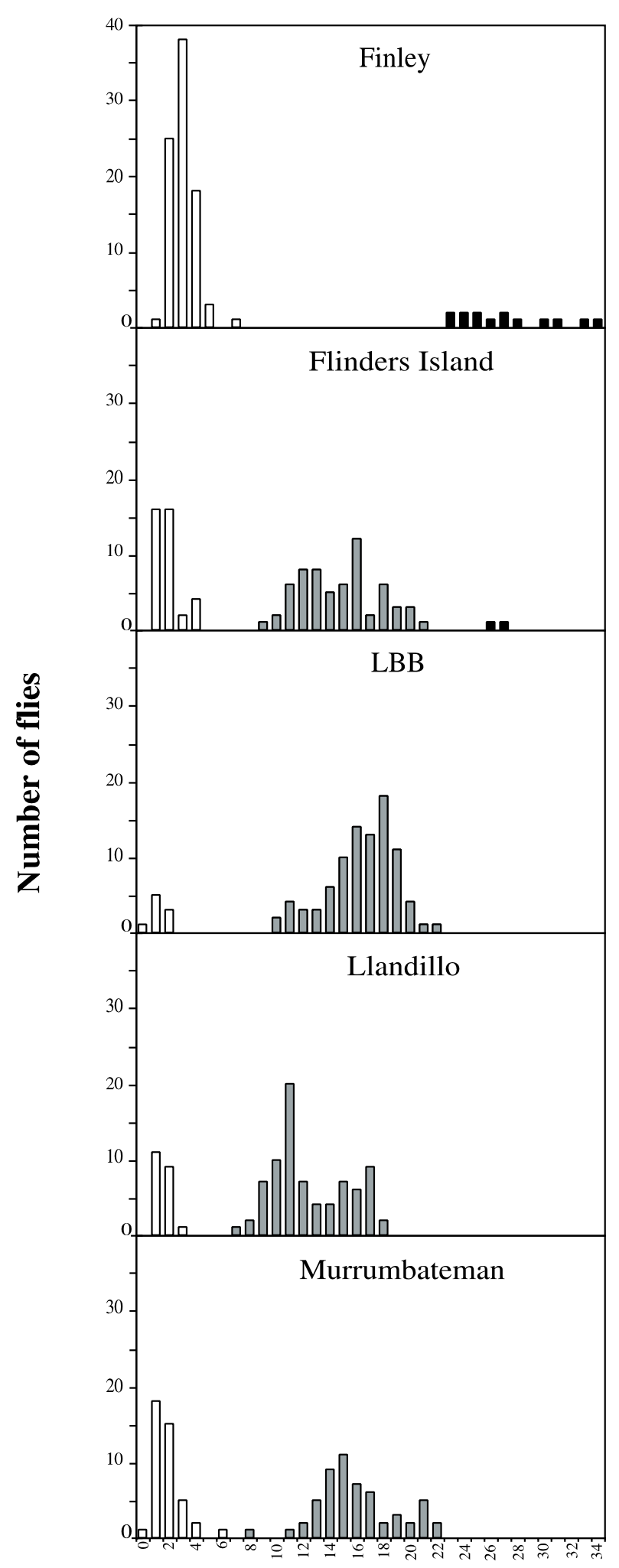

MCE activity (pmol malathion/min)
Fig. 4 The distribution of MCE activities (pmole malathion/ $\mathrm{min}$ ) for 100 individuals from each of the five mass populations of Lucilia cuprina. The numbers of individuals with low MCE activity are indicated by clear bars, intermediate MCE activity by grey bars and high MCE activity by black bars.

Table 2 Malathion and diazinon percentage mortalities and percentage low MCE phenotype for each of the five mass populations of Lucilia cuprina

\begin{tabular}{lccc}
\hline $\begin{array}{l}\text { Mass } \\
\text { population }\end{array}$ & $\begin{array}{l}\text { Malathion } \\
\text { percentage } \\
\text { mortality } \dagger\end{array}$ & $\begin{array}{c}\text { Percentage } \\
\text { low MCE } \\
\text { phenotype }\end{array}$ & $\begin{array}{c}\text { Diazinon } \\
\text { percentage } \\
\text { mortality } \dagger\end{array}$ \\
\hline Finley & $73 \pm 2.0$ & 86 & $16 \pm 1.3$ \\
Flinders Island & $96 \pm 0.4$ & 35 & $19 \pm 1.5$ \\
LBB & $92 \pm 0.8$ & 9 & $78 \pm 1.7$ \\
Llandillo & $88 \pm 1.1$ & 12 & $45 \pm 2.5$ \\
Murrumbateman & $90 \pm 0.9$ & 42 & $23 \pm 1.8$ \\
\hline
\end{tabular}

$\dagger$ Results are expressed as percentage mortality \pm binomial standard errors.

frequencies of low MCE homozygotes, as expected given that there are low/intermediate MCE heterozygotes and intermediate MCE homozygotes in these populations. However, the frequency of malathion susceptible homozygotes in the Finley population was significantly lower than the frequency of low MCE homozygotes $\left(\chi_{1}^{2}=5.22, P<0.01\right)$, suggesting that other malathion resistance mechanisms may be present in the Finley population in addition to high MCE activity.

The frequency of diazinon resistance ranged from $22 \%$ in the LBB population to $84 \%$ in the Finley population (Table 2 ).

\section{Discussion}

Our analysis of all three field-derived samples of L. cuprina revealed only the three levels of MCE activity originally described (Smyth et al., 1994). High MCE activity was almost invariably associated with malathion resistance, whereas low MCE activity was always associated with resistance to diazinon. Consequently, malathion resistance was in almost complete disequilibrium with diazinon resistance. The major exceptions are the Finley mass population, which may contain other mechanisms for malathion resistance, and the three screened isogenic lines that are resistant to both malathion and diazinon. The double resistance of these isogenic lines can be explained by a duplication of the $L c \alpha \mathrm{E} 7$ gene (see below). Taken together, these results indicate that malathion and diazinon resistances in field populations of $L$. cuprina are generally caused by the Gly ${ }^{137}-$ Asp and $\operatorname{Trp}^{251}-$ Leu variants of E3 encoded by alleles of the $L c \alpha \mathrm{E} 7$ gene. 
In the 34 unscreened isogenic lines we found all three levels of MCE activity; high, intermediate and low, albeit there was only one high MCE activity line (RM8). Only high MCE activity was found in the 20 screened isogenic lines, and these activities were all similar to the high MCE activity characterized from the RM8 unscreened isogenic line. Anticipating the difficulty of assigning MCE activity phenotypes in the five nonisogenic populations, because of the suggestion of complex dominance relationships from earlier experiments (Smyth et al., 1994), we determined the dominance relationships between the low, intermediate and high MCE activities with the unscreened isogenic lines. The MCE activity phenotypes of $F_{1}$ progeny of crosses involving various unscreened lines exhibit the same complex but consistent set of dominance relationships we previously found: no dominance between low and high MCE activities; intermediate dominant to high MCE activities; low semidominant to intermediate MCE activities. These relationships differ from the patterns of dominance among the same MCE phenotypes with respect to malathion resistance. The malathion resistance associated with high activity was semidominant to the malathion susceptibility associated with either the low or intermediate MCE activities.

Although the dominance relationships among MCE activity classes preclude a complete assignment of genotypes in the mass populations, the frequencies of MCE activities in these populations were broadly consistent with those found in the sample of unscreened isogenic lines. The variation in these frequencies among populations may reflect the heterogeneity in their collection times. Low MCE activity was most frequent in the most recently collected population, Finley, and least frequent in LBB, the earliest collected population. The Finley population also appeared to show a marked deviation from Hardy-Weinberg equilibrium as individuals within the range of high homozygotes were more frequent than those in the range of intermediate homozygotes or high and intermediate heterozygotes.

In order to determine if malathion resistance is generally caused by high MCE activity in field populations, we tested the resistance status of all three fieldderived samples, and found that resistance to malathion was completely correlated with MCE activities in both sets of the isogenic lines. All low and intermediate MCE activity lines were susceptible to malathion, whereas the high MCE activity lines were resistant. The frequencies of malathion resistance in the five mass populations are also generally consistent with the data on MCE activities in these populations, and malathion resistance in prior surveys of field populations (Hughes et al., 1984). The exception is the Finley population for which the frequency of malathion susceptibility was lower than expected from the MCE activity data. This finding is consistent with the evidence that intermediate MCE activity is not common in this population. One possible explanation for the skewed distribution of MCE activities in the Finley population is that a different or second genetic basis for high MCE activity may be present, which may also underlie the apparent deviation from Hardy-Weinberg. Another possibility is that additional resistance mechanisms may contribute to malathion resistance in this population. Altered acetylcholinesterase (AChE) and enhanced glutathione S-transferase and mixed function oxidase activities have been shown to confer resistance to malathion in other pest insect species (Takahashi \& Yasutomi, 1987; Bull \& Pryor, 1990; Scott et al., 1990), and one or more of these mechanisms may be present in the Finley population.

Diazinon resistance is also conferred by a mutation in the LcaE7 gene. An amino acid substitution at position 137 results in increased OP hydrolase and decreased carboxylesterase activities (Newcomb et al., 1997; Campbell et al., 1998a). We sought to determine whether resistance to both malathion and diazinon could be conferred by the OP hydrolase and high MCE activities encoded by alleles of the $L c a E 7$ gene. Diazinon resistance was in complete disequilibrium with malathion resistance in the unscreened isogenic lines tested. The high MCE line was resistant to malathion and all low MCE lines were resistant to diazinon. These lines were also assayed for OP hydrolase activity by scoring for the presence of E3 activity on native PAGE (data not shown), and all the low MCE activity lines lacked any visible E3 staining activity, indicating the presence of the OP hydrolase. Therefore, the diazinon resistance in the low MCE activity lines was consistent with expectations based on the OP hydrolase activity encoded by the Gly ${ }^{137}$-Asp substitution in the LcaE7 gene.

Only one mass population may contain some flies resistant to both malathion and diazinon. The combined frequencies of malathion and diazinon resistances in the Finley population exceeded $100 \%$ (Table 2). We have already noted that this population may contain other malathion resistance mechanisms, such as altered AChE, that have also been shown to contribute resistance to diazinon (Fournier et al., 1992; Waters et al., 1992; Mutero et al., 1994). Thus, with the exception of the Finley population, the frequencies of malathion and diazinon resistances were consistent with the resistances being in disequilibrium in the mass populations. The data for malathion and diazinon resistances also support prior evidence for high frequencies of diazinon resistance and low frequencies of malathion resistance in the field (Hughes et al., 1984; Levot, 1995).

In contrast to the unscreened isogenic lines, malathion and diazinon resistances were not in complete 
disequilibrium in the screened isogenic lines. Three of the nine lines tested were resistant to both compounds. Our preliminary analysis of genetic variation around the $L c \alpha \mathrm{E} 7$ gene using restriction fragment length polymorphisms has revealed seven different haplotype classes, each one associated exclusively with (i) OP susceptibility (classes A, B and C) (ii) malathion resistance (E and F) or (iii) diazinon resistance (D and G; T.M.B., K.A.S. and J.G.O., unpubl. data). The restriction patterns suggest that two of the three screened isogenic lines resistant to both malathion and diazinon have duplicated $L c \alpha \mathrm{E} 7$ genes consistent with their resistance status. Tooronga 2.2 has both the D and E haplotype classes and 60NE 3.1 has classes $F$ and $G$. The third line has not been characterized. Therefore, for an isogenic fly to be resistant to both malathion and diazinon it appears that it must carry two different $L c \alpha \mathrm{E} 7$ genes, one presumptively encoding E3 Gly ${ }^{137}$-Asp and the other presumptively E3 Trp ${ }^{251}$-Leu.

We have found, with a few notable exceptions, that high MCE activity confers almost all malathion resistance in these three field-derived samples, and on this basis we conclude that the $\operatorname{Trp}^{251}$-Leu amino acid substitution in the $L c \alpha \mathrm{E} 7$ gene is responsible for most malathion resistance in field populations of L. cuprina. We also report that double resistance to malathion and diazinon can occur and appears to involve a duplication of the region containing the Lc $\alpha \mathrm{E} 7$ gene. Double resistance is not common in the field, malathion resistance is about $11 \%$ and one-third of those chromosomes may also carry diazinon resistance. Restrictions on the rate of recombination or fitness costs associated with the duplications may account for the low frequency of these doubly resistant chromosomes.

\section{Acknowledgements}

The authors would like to thank Gary Levot and Narelle Sales (NSW Department of Agriculture) for providing many of the stocks used to generate the isogenic lines. We would also like to thank Peter Campbell and the members of the esterase and blowfly groups at CSIRO Division of Entomology for their helpful discussions and comments on the manuscript.

\section{References}

BRADFORD, M. 1976. A rapid and sensitive method for the quantitation of microgram quantities of protein utilising the principle of protein-dye binding. Analyt. Biochem., 72, 248-254.

BULL, D. L. AND PRYOR, N. W. 1990. Characteristics of resistance in house flies subjected to long-term concurrent selection with malathion and permethrin. Pestic. Biochem. Physiol., 37, 101-115.

CAMPBell, P. M., TROTT, J. F., ClAUdiANOS, C., SMYTH, K. A., RUSSELL, R. J. AND OAKESHOTT, J. G. 1997. Biochemistry of esterases associated with organophosphate resistance in Lucilia cuprina, with comparisons to putative orthologues in other Diptera. Biochem. Genet., 35, 17-40.

CAMPBELL, P. M., NEWCOMB, R. D., RUSSELL, R. J. AND OAKESHOTt, J. G. 1998a. Two different amino acid substitutions in the ali-esterase, E3, confer alternative forms of organophosphorus insecticide resistance in the Australian sheep blowfly, Lucilia cuprina. Insect Biochem. Mol. Biol., 28, 139-150.

CAMPBELL, P. M., YEN, J. L., MASOUMI, A., RUSSELL, R. J., BATTERHAM, P., MCKENZIE, J. A. AND OAKESHOTT, J. G. 1998b. Crossresistance patterns among Lucilia cuprina (Diptera: Calliphoridae), resistant to organophosphorus insecticides. J. Econ. Entomol., 91, 367-375.

DUNCAN, D. B. 1975. $t$ tests and intervals for comparisons suggested by the data. Biometrics, 31, 339-350.

FINNEY, D. J. 1971. Probit Analysis. Cambridge University Press, Cambridge.

FOSTER, G. G., WHITTEN, M. J., VOGT, W. G., WOODBURN, T. L. AND ARNOLD, J. T. 1978. Larval release method for genetic control of the Australian sheep blowfly, Lucilia cuprina (Wiedemann) (Diptera: Calliphoridae). Bull. Ent. Res., 68, 75-83.

FOURNIER, D., BRIDE, J. M., POIRIE, M., BERGÉ, J.-B. AND PLAPP, F. W. 1992. Insect glutathione $S$-transferases. J. Biol. Chem., 267, 1840-1845.

HUGHES, P. B. 1981. Spectrum of cross-resistance to insecticides in field samples of the primary sheep blowfly, Lucilia cuprina. Int. J. Parasitol., 11, 475-479.

HUGHES, P. B., GREEN, P. E. AND REICHMANN, K. G. 1984. Specific resistance to malathion in laboratory and field populations of the Australian sheep blowfly, Lucilia cuprina (Diptera: Calliphoridae). J. Econ. Entomol., 77, 1400-1404.

LEVOT, G. W. 1995. Resistance and control of sheep ectoparasites. Int. J. Parasitol., 25, 1355-1362.

MCKENZIE, J. A., DEARN, J. M. AND WHITTEN, M. J. 1980. Genetic basis of resistance to diazinon in Victorian populations of the Australian sheep blowfly, Lucilia cuprina. Aust. J. Biol. Sci., 33, 85-95.

MCKenZie, J. A., PARker, A. P. AND Yen, J. L. 1992. Polygenic and single gene responses to selection for resistance to diazinon in Lucilia cuprina. Genetics, 130, 613-620.

MUtero, A., PRAlAvorio, M., BRIDE, J.-M. AND FOURNIER, D. 1994. Resistance-associated point mutations in insecticideinsensitive acetylcholinesterase. Proc. Natl. Acad. Sci. U.S.A., 91, 5922-5926.

NeWCOMB, R. D., CAMPBell, P. M., Ollis, D. L., CHEAH, E., RUSSELL, R. J. AND OAKESHOTT, J. G. 1997. A single amino acid substitution converts a carboxylesterase to an OP hydrolase to confer organophosphorus (OP) insecticide resistance in the sheep blowfly, Lucilia cuprina. Proc. Natl. Acad. Sci. U.S.A., 94, 7464-7468.

NORRIS, K. R. 1990. Evidence for the multiple exotic origin of Australian populations of the sheep blowfly, Lucilia cuprina 
(Wiedemann) (Diptera: Calliphoridae). Aust. J. Zool., 38, 635-648.

RAFTOS, D. A. 1986. The biochemical basis of malathion resistance in the sheep blowfly. Pestic. Biochem. Physiol., 26, 302-309.

SAS, 1988. Additional SAS/STAT TM Procedures. Technical Report P-179 release 6.03. SAS Institute Inc, Cary, NC.

SCOTT, J. G., COCHRAN, D. G. AND SIEGFRIED, B. D. 1990. Insecticide toxicity, synergism, and resistance in the German cockroach (Dictyoptera: Blattellidae). J. Econ. Entomol., 83, 1698-1703. SHANAHAN, G. J. AND HART, R. J. 1966. Change in response of Lucilia cuprina Weid. to organophosphorus insecticides in Australia. Nature, 212, 1466-1467.

SMYTH, K. A., RUSSEll, R. J. AND OAKeshott, J. G. 1994. A cluster of at least three esterase genes in Lucilia cuprina includes malathion carboxylesterase and two other esterase genes implicated in resistance to organophosphates. Biochem. Genet., 32, 437-453.
SMYTH, K. A., WALKER, V. K., RUSSELL, R. J. AND OAKESHOTT, J. G. 1996. Biochemical and physiological differences in the malathion carboxylesterase activities of malathion susceptible and resistant lines of the sheep blowfly, Lucilia cuprina. Pestic. Biochem. Physiol., 54, 48-55.

TAKAHASHI, M. AND YASUTOMI, K. 1987. Insecticidal resistance of Culex tritaeniorhynchus (Diptera: Culicidae) in Japan: Genetics and mechanisms of resistance to organophosphorus insecticides. J. Med. Entomol., 24, 595-603.

WATERS, L. C., SHAW, B. J. AND CH'ANG, L. Y. 1992. Regulation of the gene for Drosophila P450-B1, a P450 isozyme associated with insecticide resistance. In: Mullin, C. A. and Scott, J. G. (eds) Molecular Mechanisms of Insecticide Resistance, pp. 41-52. ACS Symposium Series 505. American Chemical Society, Washington, DC.

WHYARD, S., RUSSELL, R. AND WALKER, V. K. 1994. Insecticide resistance and malathion carboxylesterase in the sheep blowfly, Lucilia cuprina. Biochem. Genet., 32, 9-24. 